\begin{tabular}{|l|c|c|c|}
\hline Eiszeitalter u. Gegenwart & $\mathbf{2 7}$ & $\begin{array}{c}75-81 \\
\text { 5 Abb., 1 Tab. }\end{array}$ & Öbringen/Württ. 1976 \\
\hline
\end{tabular}

\title{
Die weichseleiszeitliche Eisrandlage im Osten von Hamburg
}

\author{
Dieter JaschKe *)
}

Continental glacier, ice sheet, melting water, erosion, "Tunneltal“, till

Kurzfassung: Gegen die herrschende Lehrmeinung wird die Ansicht vertreten, daß das weichseleiszeitliche Inlandeis östlich Hamburgs über die postulierte maximale Eisrandlage der A-1Linie hinaus in Richtung Süden vorgestoßen ist. Zumindest hatte es sich während des Hochglazials bis in den heutigen Elberaum hinein ausgedehnt. Nach dem Rückschmelzen des Eisrandes bis zur A-1-Linie blieb ein Gletscher, der mit dem Inlandeis weiterhin Verbindung hatte, im Bereich des heutigen Billetales zurück. Seine Schmelzwässer räumten entlang des Urstromtalhanges zwischen Bergedorf und Geesthacht stark versteilte Kerbtäler aus und brachten subglazial das Tunneltal der Bille hervor. Wichtigstes stratigraphisches Indiz für die neue Hypothese ist im Billetal eine zwischen zwei Schmelzwasserschichten eingeschaltete Moränenlage.

[The Position of the Ice Sheet in Weichsel Time east of Hamburg]

A bstract: Contrary to the prevailing opinion the author demonstrates that in the eastern part of the Hamburg Region the continental glacier of the Weichsel Glaciation advanced beyond the A-1-Line, being generally accepted as the marginal position of the ice sheet. At the culminating point of the glaciation the glacier at least expanded as far as the present Elbe Valley. After the recession of the ice sheet to the A-1-Line a glacier tongue remained in the area of the actual Bille Valley, being still in connection with the continental glacier. The melting water of this lobe eroded steep V-shaped valleys into the slope of the Elbe "Urstromtal" between the cities of Geesthacht und Bergedorf. At the same time the ice and its melting water subglacially eroded the "Tunneltal" of the Bille Valley. The most important evidence of this hypothesis is a layer of till between deposits of subglacial streams on the valley bottom.

Nach anerkannter Lehrmeinung (GRIPp 1964, WoldSTEDT 1975) hat sich das weichselzeitliche Inlandeis östlich von Hamburg nicht über die morphographisch kartierte Linie der A-1-Endmoräne des Trittauer Raumes ausgedehnt. Eine Reihe gegenteiliger Ansichten sind vor und nach Formulierung dieser These geäußert worden. Bereits die geologischen Kartenblätter, die für dieses Gebiet von der Preußischen Geologischen Landesaufnahme zwischen 1905 und 1926 erstellt wurden, gehen bei der Datierung der Formationen von einem weichseleiszeitlichen Eisvorstoß aus, der bis weit über den heutigen Elberaum hinaus nach Süden reichte. Andere Autoren, die in der Lüneburger Heide glazial-morphologischen Fragestellungen nachgegangen sind, deuten ebenfalls an, daß möglicherweise weichselzeitliches Eis an der Formengestaltung zumindest der Nordheide beteiligt war (STAPPENBeCK 1905, Olbricht 1909, Stoller 1914). Als Indizien werden von geologischer Seite vor allem die Ergebnisse petrographischer Sedimentanalysen angeführt. Der hohe Kalkgehalt der oberflächennah anstehenden Sedimente des "Altmoränengebietes“ zwischen Elbeurstromtal und angenommenem Eisrand läßt auf eine geringe Alterung des Substrats schließen (Tab. 1). Aber auch in morphologischer Hinsicht gibt es Indizien, die auf einen südwärtigen Eisvorstoß hinweisen, der über die postulierte Grenze hinausgeht.

*) Anschrift des Verfassers: Dr. D. J a s ch ke, Institut für Geographie und Wirtschaftsgeographie, Bundesstr. 55, 2000 Hamburg 13. 
Tab. 1: Kalkgehalt glaziärer Sedimente im Osten von Hamburg

\begin{tabular}{lclc}
\hline Aufschluß & $\begin{array}{c}\text { Tiefe der } \\
\text { Entnahme }\end{array}$ & Sediment & $\begin{array}{l}\text { Kohlensaurer Kalk } \\
\text { im Feinboden < 2 mm }\end{array}$ \\
\hline Ziegeleigrube Schwarzenbek & $2,0 \mathrm{~m}$ & Geschiebemergel & $4,2 \%$ \\
Lehmgrube bei Volksdorf & $1,2 \mathrm{~m}$ & Mergel & $4,6 \%$ \\
Ziegeleigrube Börnsen & $2,0 \mathrm{~m}$ & Mergelsand & $4,6 \%$ \\
Mergelgrube E Hornbek & $1,0 \mathrm{~m}$ & Geschiebemergel & $6,8 \%$ \\
Tongrube W Tesperhude & $1,2 \mathrm{~m}$ & Tonmergel & $7,2 \%$ \\
Tongrube in Tesperhude & $1,3 \mathrm{~m}$ & Tonmergel & $7,6 \%$ \\
Mergelgrube E Grabau & $1,2 \mathrm{~m}$ & Mergel & $8,6 \%$ \\
Mergelgrube N Gülzow & $1,5 \mathrm{~m}$ & Geschiebemergel & $8,7 \%$ \\
Mergelgrube SW Poppenbüttel & $1,0 \mathrm{~m}$ & Geschiebemergel & $9,5 \%$ \\
Mergelgrube 2 km SW Tramm & $0,3 \mathrm{~m}$ & Tonmergel & $14,9 \%$ \\
Mergelgrube in Kollow & $1,0 \mathrm{~m}$ & Geschiebemergel & $18,9 \%$ \\
Mergelgrube N Kollow & $1,2 \mathrm{~m}$ & Mergel & $19,7 \%$ \\
Mergelgrube W Kollow & $1,0 \mathrm{~m}$ & Geschiebemergel & $41,1 \%$ \\
\hline
\end{tabular}

Quellen: Erläuterungen zur Geologischen Karte von Preußen und benachbarten Bundesstaaten. Hrsg. von der Königlich Preußischen Geologischen Landesanstalt.

Lieferung 168 - Blatt Schwarzenbek, Berlin 1911, Blatt Hamwarde, Berlin 1911,

Lieferung 176 - Blatt Bergedorf, Berlin 1912, Blatt Glinde, Berlin 1913.

Dazu gehören die heute zum Teil trockenen Kerbtäler, die den Steilabfall auf der Nordseite des Elbeurstromtals zwischen Bille- und Stecknitzmündung auf kurzer Distanz mit großem Gefälle überwinden (Abb. 1 u. 2).

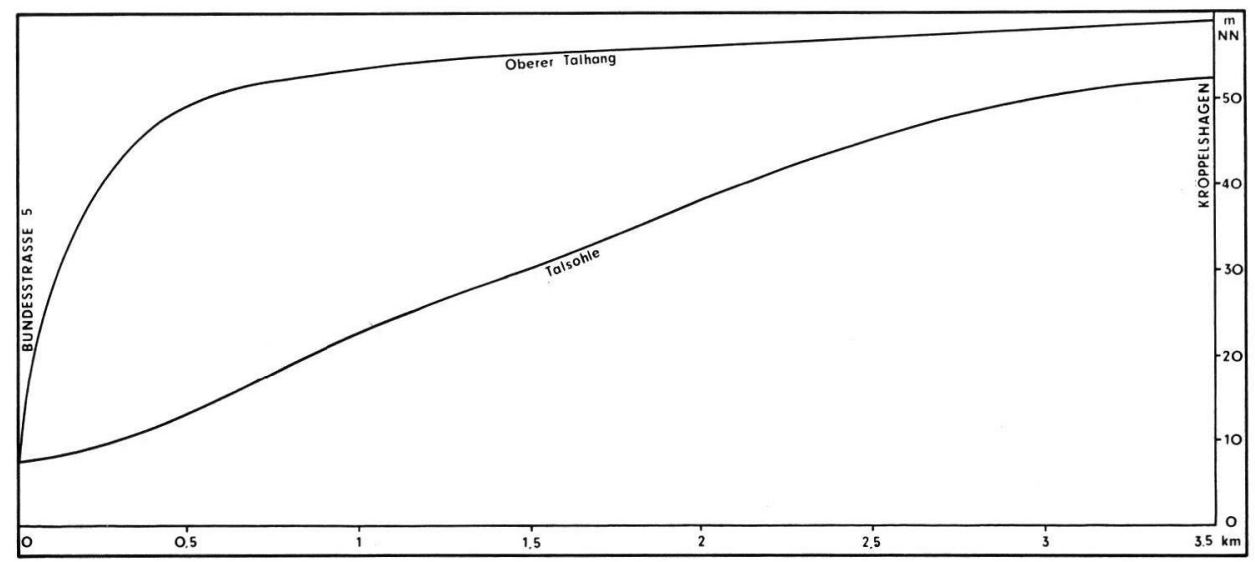

Abb. 1: Schematisches Längsprofil des Dallbektales.

Das Flußlängsprofil ist auf eine erheblich tiefere Elbtalsohle als die jetzige eingeregelt. Die Täler müssen erodiert worden sein, als die Sohle des Urstromtals als lokale Erosionsbasis während des weichselzeitlichen Hochglazials, bzw. späten Hochglazials, $20-25 \mathrm{~m}$ unter dem heutigen Niveau lag. Gegen eine holozäne Genese sprechen nicht nur die Anlage des Längsprofils und der trockene Zustand mancher Täler, sondern auch die geringe Wasserführung der Bäche, deren rezente Erosionsleistungen eine Ausräumung in der relativ kurzen Postglazialzeit ausschließen. Zur Ausgestaltung jener Täler müssen größere Was- 
sermassen beigetragen haben, als sie für die Nacheiszeit denkbar sind. In Frage kommen also nur Schmelzwässer von Gletschern, die unmittelbar nördlich des Urstromtales lagen. Eine Wasserzufuhr aus dem Bereich der postulierten maximalen Eisrandlage scheidet aus, da das Billetal, das nach Ansicht des Autors im übrigen nach einer weiteren Nordverlagerung des Gletscherrandes angelegt wurde, die Schmelzwässer aufnahm. Solifluidale Prozesse unterstützten den Vorgang der Talbildung, insbesondere durch denudativen Hangabtrag; die Schmelzwässer waren es aber, die über ausreichend Energie verfügten, um das abgerutschte Material zu verfrachten und obendrein linear in die Tiefe zu erodieren, so $\mathrm{da}$ der typische Kerbtalcharakter jener Hohlformen sich einstellen konnte. Weitere Indizien für die These, daß sich das weichselzeitliche Eis während des Hochglazials über die Linie der von GRIPP kartierten A-1-Moränen hinaus nach Süden ausgedehnt hat, lassen sich sowohl im morphographischen als auch stratigraphischen Sinne im Bereich des unteren Billetals anführen. Es kann der gängigen Theorie zugestimmt werden, daß der obere Teil des Billetales als Tunneltal angelegt worden ist. Hier befand sich das weichselzeitliche Inlandeis für einen längeren Zeitraum in stationärer Lage: nach der Lehrmeinung in seiner äußersten Süderstreckung, nach neuerer Auffassung innerhalb eines Rückzugsstadiums. Vieles spricht dafür, daß auch der untere Talverlauf, und zwar bis zur Einmündung in das Elbeurstromtal ursprünglich eine subglaziale Ausgestaltung erfahren hat. Innerhalb der von Nordost nach Südwest gerichteten Furche, die heute von der Bille durchflossen wird und die bereits präweichsel vorgezeichnet gewesen sein wird, lagerte ein flacher Eislobus als Rest der ehedem größeren und inzwischen niedergetauten Inlandeismasse. Im Nordosten fand die Gletscherzunge Anschluß an den sich regenerierenden Eisrand. Sehr wahrscheinlich sind die Schmelzwässer, die nicht der subglazialen Hauptentwässerungsrichtung folgten, sondern über die heutige Wasserscheide hinaus nach Süden abflossen, identisch mit den Gletscherbächen, die die gegen das Urstromtal gerichteten Kerbtäler, zumindest im Bereich zwischen Bergedorf und Geesthacht, hervorgebracht haben.

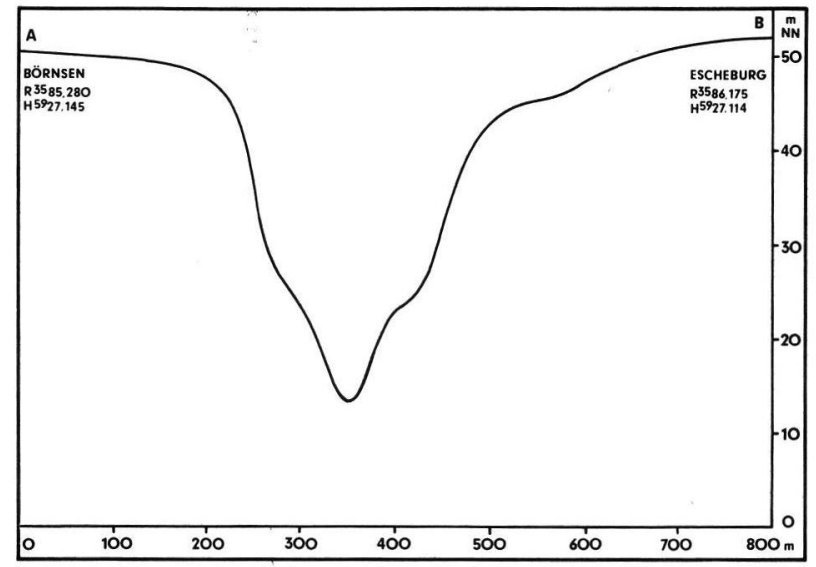

Abb. 2: Schematisches Querprofil des Dallbektales.

Der Beweis für diẹ aufgestellten Thesen läßt sich am anschaulichsten am Beispiel der morphogenetischen Entwicklung des Billetales führen (Abb. 3 u. 4).

Die Talung gestaltet sich heute im Flußunterlauf folgendermaßen: In der Höhe Reinbeks hat das Tal - bezogen auf die Ausdehnung zwischen den oberen Rändern - eine Breite von $1-2 \mathrm{~km}$. Die Sohle, die $30-40 \mathrm{~m}$ unter dem Niveau der Umgebung liegt, mißt eine Breite von $500-1000 \mathrm{~m}$. Der Lauf des Tales weist zahlreiche Windungen auf, 
die Winkel bis zu $90^{\circ}$ beschreiben. Sanft geböschte Gleithänge lösen sich mit stark versteilten Prallhängen ab. Innerhalb dieser weiten Talung mäandriert der rezente Fluß zwischen den Rändern eines Terrassensystems, das den Auebereich auf 100 bis $200 \mathrm{~m}$ Breite einengt. Das Talquerprofil zeigt danach eine Abfolge von

- den 40 bis 50 m über NN liegenden Rahmenhöhen, die den Charakter einer schwach gewellten Grundmoränenlandschaft aufweisen,

- über eine steile Prallhangpartie auf der einen Seite und einen allmählich abdachenden Gleithang auf der anderen Seite

- zu den fast ebenen oder nur gering zum Flusse geneigten Terrassen,

— in die die Aue mit deutlichem Steilabfall eingesenkt ist.

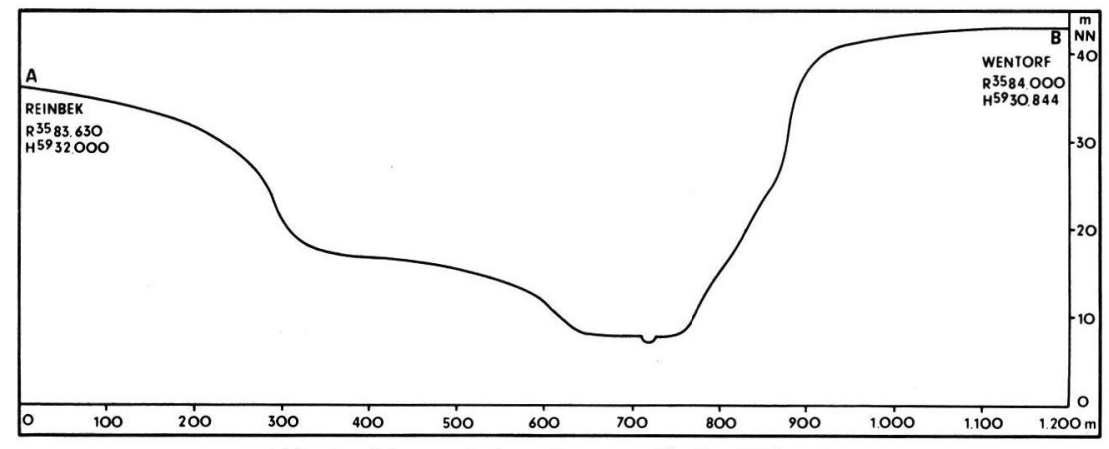

Abb. 3: Schematisches Querprofil des Billetales.

Im Verlauf des Längsprofils konvergieren die Terrassen mit der Aue in der Gefällsrichtung des Tales: In Höhe des Krabbenkamps zwischen Wohltorf und Aumühle liegen die Terrassen noch etwa 7,5 m über der Wiesensohle, im Südwesten von Reinbek beträgt die Sprunghöhe nur noch $1 \mathrm{~m}$ und kurz vor Einmündung der Bille in das Elbeurstromtal verschwinden die Terrassen gänzlich unter den Auesedimenten, die bereits mit den Marschenablagerungen des ehedem amphibischen Stromspaltungsgebietes identisch sind.

Daraus läßt sich folgern, daß das Gefälle der Flußaue auf die lokale Erosionsbasis der rezenten Elbe eingeregelt ist, während die steiler einfallenden Terrassen, die Relikte eines älteren Talbodens, mit einem niedrigeren Erosionsniveau in Beziehung standen.

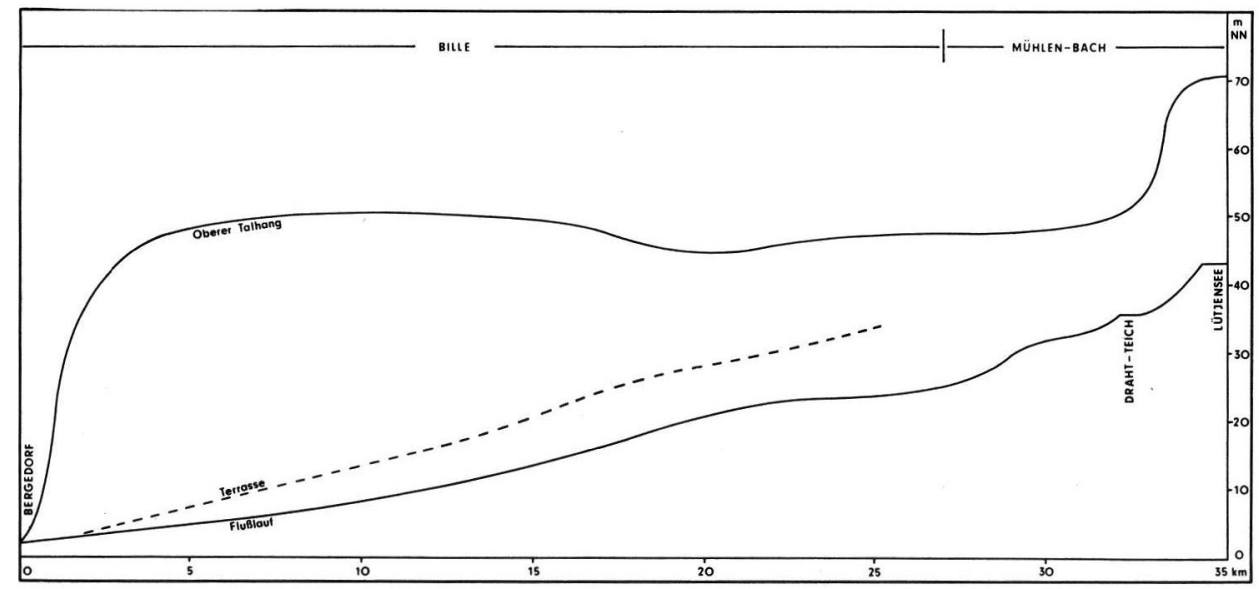

Abb. 4: Schematisches Längsprofil des Billetales. 
Die heutige morphographische Gestalt des Billetales geht im wesentlichen auf vier genetische Phasen zurück:

\section{Phase 1}

Während des weichselzeitlichen Hochglazials brachten subglaziale Ausräumungsprozesse unterhalb des postulierten Billelobus das Tunneltal der "Urbille“ hervor. Da der Gletscher bis zu dem inzwischen auf $-20 \mathrm{~m}$ NN erodierten Urstromtal der Elbe herabreichte, lag die Basis des Tunneltals beträchtlich unter dem Niveau des heutigen Talbodens. In der Höhe Reinbeks war das Gletscherbett bis zu 20 m tief in die Sedimente des Miozäns abgesenkt. Stratigraphische Untersuchungen haben gezeigt (Abb.5), daß sich über der tertiären Abtragungssohle eine maximal 1-1,5 m mächtige Packung geschichteter Feinund Mittelsande befindet, in die allerdings auch größere Blöcke eingelagert sind. Diese Ablagerung, die deutlich kreuzgeschichtet ist, keine gleichbleibende Mächtigkeit aufweist und verschiedentlich völlig zurücktritt, markiert im Subglazialbereich den Übergang von der Erosion zur Akkumulation.

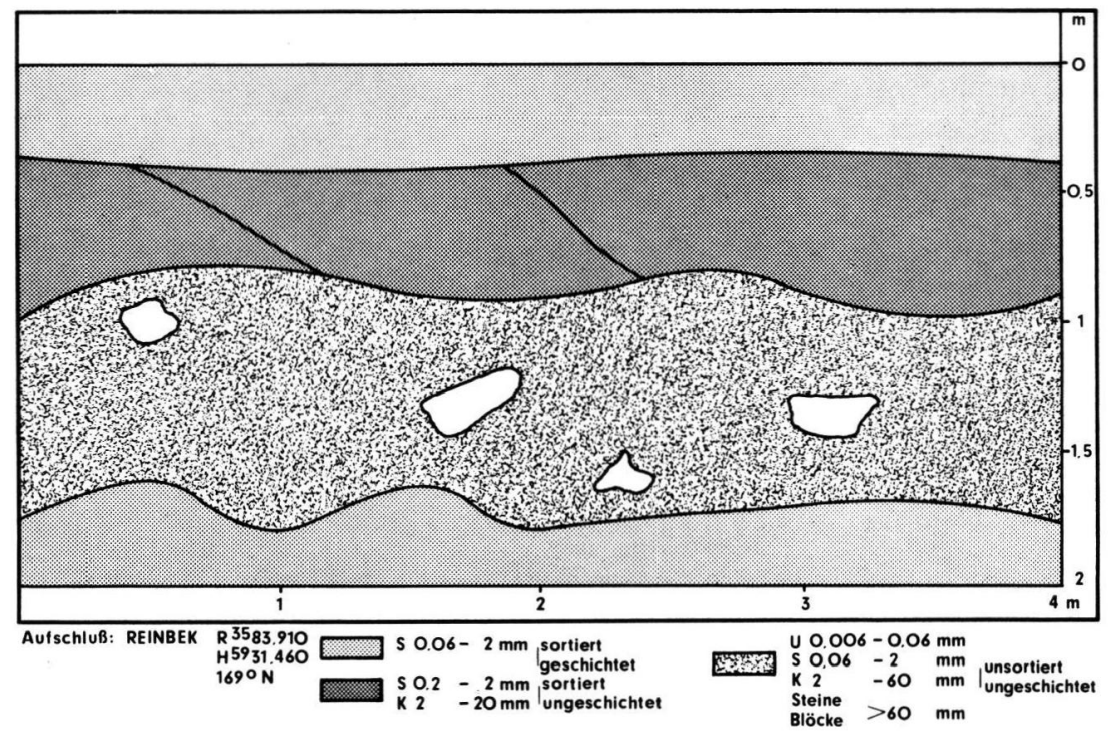

Abb. 5: Stratigraphisches Profil der Billetal-Terrasse.

\section{Phase 2}

Die sich anschließende Phase, die zeitlich in das späte Hochglazial fällt, ist charakterisiert durch den Absatz einer 1,5-2 m mächtigen Moräne, die das niedertauende Gletschereis freigab. Innerhalb einer aus Schluff und Sand bestehenden Matrix, in die vereinzelt Linsen geschichteter Sande eingeschaltet sind, schwimmen Geschiebe von maximal 1,5 m Durchmesser. Der Einwand, daß es sich hier auch um solifluidale Rutschmassen handeln könnte, wird entkräftet durch das fehlende Gefälle der Basisfläche. Das Moränenmaterial ist wiederum von geringmächtigen geschichteten Sanden eingedeckt, die sich petrographisch nur unwesentlich von den Sanden im Liegenden unterscheiden. Ihre Schüttung erfolgte durch Schmelzwässer, die sich bei und nach Abschmelzen des Billelobus von Norden und Nordosten her in das als Sammler fungierende Billetal ergossen. Alle drei Sedimentkörper sind parallel gelagert und fallen gegen das Elbetal unter gleichem Winkel ein. Daraus ist 
zu schließen, daß es innerhalb des Sedimentationszeitraumes keinen nennenswerten Anstieg der lokalen Erosionsbasis gegeben hat. Die Neigungsverhältnisse des damaligen Schmelzwasserstromes werden durch das heutige Terrassensystem angezeigt, das als Relikt jener alten Talsohle zu verstehen ist.

\section{Phase 3}

Die Zerschneidung des Talbodens, die bis zu den liegenden Sanden herabreicht, erfolgte im Spätglazial, als der Eisrand beträchtlich weiter im Norden lag und der ehemalige Periglazialraum bereits einen flächendeckenden Vegetationsmantel trug. Obwohl der Meeresspiegel und damit auch die lokale Erosionsbasis des Elbetals infolge des weltweiten Eisniedertaus in stetiger Hebung begriffen waren, begannen die Schmelzwässer erneut linear zu erodieren, da der größte Teil der mitgeführten Flußfracht schon im Vorfeld des Gletschers abgesetzt worden war und damit die Unterläufe der Schmelzwässer einen Überhang kinetischer Energie besaßen. Damit entstand ein System von Erosionsterrassen, das aber nicht länger im Sinne Pfefferles (1935) als Talsandterrassensystem interpretiert werden darf. Wie die stratigraphische Beschreibung gezeigt hat, sind Talsande nur in geringer Mächtigkeit nachweisbar. Im wesentlichen werden die Terrassenkörper aus dem Material der zwischen die Schmelzwassersedimente eingeschalteten Moräne aufgebaut.

\section{Phase 4}

Im Postglazial stellte sich mit dem Einpendeln der Erosionsbasis auf das heutige Niveau kurzfristig ein Gleichgewicht zwischen Abtrag und Ablagerung ein. Eine verstärkte Akkumulation zwischen den Terrassenrändern setzte ein, als in historischer Zeit mit der Rodung der natürlichen Waldbedeckung weite Teile der Erdoberfläche ungeschützt der Abtragung ausgesetzt wurden, so daß sich vor allem bei Starkregen oder zur Zeit der Schneeschmelze ein erheblicher Abtransport feiner Bodenbestandteile einstellte, die im Auebereich insbesondere bei saisonaler Überschwemmung abgelagert wurden. Diese Vorgänge dauern bis zur Jetztzeit an.

Anhand morphographischer und stratigraphischer Merkmale hat sich der genetische Hergang der Oberflächengestaltung für den Bereich des Billetales aufzeigen lassen. Implizit steckt in dieser Darstellung der Beweis für die These, daß das weichselzeitliche Inlandeis über die bisher angenommenen Grenzen hinaus nach Süden vorgestołen ist.

\section{Schriftenverzeichnis}

GrimmeL, E. (1971): Geomorphologische Untersuchungen in der nordöstlichen Lüneburger Heide. - Hamburger Geogr. Studien, 27; 57 S., 10 Abb., 4 Karten; Hamburg (Hirt).

GripP, K. (1924): Über die äußerste Grenze der letzten Vereisung in Nordwestdeutschland. Mitt. Geogr. Ges. in Hamburg, 36: 159-245, Hamburg.

- (1933): Geologie von Hamburg und seiner näheren und weiteren Umgebung. - 154 S., 35 Abb., 16 Tafeln; Hamburg (Gesellschaft der Freunde des Vaterländischen Schul- und Erziehungswesens).

- (1964): Erdgeschichte von Schleswig-Holstein. - 411 S., 63 Abb., 11 Tab., 57 Tafeln, 3 Karten; Neumünster (Wachholtz).

Illies, H. (1952): Die eiszeitliche Fluß- und Formengeschichte des Unterelbe-Gebietes. - Geol. Jb., 66: 525-558; Hannover.

- (1955): Die Vereisungsgrenzen in der weiteren Umgebung Hamburgs, ihre Kartierung und stratigraphische Bewertung. - Mitt. Geogr. Ges. in Hamburg, 51: 7-54; Hamburg.

Königlich Preussische Geologische Landesanstalt: Erläuterungen zur Geologischen Karte von Preußen und benachbarten Bundesstaaten 1:25000. Lieferung 168: Blatt Schwarzenbek und Blatt Hamwarde; Lieferung 176: Blatt Bergedorf und Blatt Glinde. - Berlin 1911-1913.

LürtiG, G. (1968): Ist die Reliefenergie ein Maß für das Alter der Endmoränen? - Eiszeitalter u. Gegenwart, 19: 197-202; Ohringen (Rau). 
OlbRicht, K. (1909): Grundlinien einer Landeskunde der Lüneburger Heide. — Forsch. z. dt. Landes- u. Volkskde., 18: 501-647; Stuttgart.

Pfefferle, R. (1935): Zur Morphologie der Talsandterrassentäler in der Umgebung von Hamburg. - 77 S., 5 Tafeln; Würzburg (Triltsch).

Richter, K. (1937): Die Eiszeit in Norddeutschland. - 179 S., 63 Abb.; Berlin (Borntraeger).

Schroeder-Lanz, H. (1964): Morphologie des Estetales. - Hamburger Geogr. Studien, 18; Hamburg.

Stappenbeck, R. (1905): Die osthannoversche Kiesmoränenlandschaft. - Z. dt. geol. Ges., 57: 52-73; Berlin.

Stoller, J. (1914): Der jungdiluviale Lüneburger Eisvorstoß. - Jber. Niedersächs. geol. Ver., 7: 214-230; Hannover.

Woldstedt, P. (1954): Saaleeiszeit, Warthestadium und Weichseleiszeit in Norddeutschland. Eiszeitalter u. Gegenwart, 4/5: 34-48; O'hringen (Rau).

- (1974): Norddeutschland und angrenzende Gebiete im Eiszeitalter. - 3. Aufl.: 500 S., 91 Abb., 26 Tab.; bearb. u. hrsg. v. K. Duphorn, Stuttgart (K. F. Koehler).

Manuskript eingeg. 12. 1. 1976. 